\title{
THE FORMATION OF SHELLS IN THE WIND FROM P CYGNI
}

\author{
F. D. KAHN \\ Department of Astronomy \\ The University \\ Manchester M13 9PL \\ England
}

\begin{abstract}
A mechanism is proposed for the formation of shells around P Cygni. These shells seem to arise at approximately two monthly intervals and each appears to undergo a steady acceleration of some $0.6 \mathrm{~cm} \mathrm{~s}^{-2}$ during the six months that it is present. In the process to be described the formation of the shells takes place in a stellar wind that moves outwards ballistically from the photosphere. Small fluctuations in wind speed are introduced there and eventually lead to the condensation of shells at a radial distance of the order of $10^{14} \mathrm{~cm}$. The density in the shells is large enough to account for their acceleration by the Lyman continuum emitted by the star. It is also proposed here that the observed time scale of two months is set by the dynamics of the flow field in interaction with the random fluctuations imposed on it at the photosphere.
\end{abstract}

\section{Introduction}

In his invited review given at the Lunteren meeting two years ago Lamers (1987) describes the characteristic variability of LBV stars and also gives an account of the episodic formation of shells around $P$ Cygni. One curious feature of the process is immediately apparent. The currently observed fluctuations in visual magnitude have an amplitude of a few per cent and appear to show no preferred period. The intervals between the appearance of successive shells are also rather irregular, but they do show a preference for a period of about two months. An even more significant difference is that the variations in magnitude are most probably due to relatively small changes in the photospheric radius and can be described as a small perturbation superposed on a star in a steady state; the formation of a shell in a wind is however a grossly non-linear effect. The intention in this paper is to establish the nature of the relation between the variability of the star and the formation of shells in its wind.

The variations in visual magnitude have not always been small. Some four centuries ago $P$ Cygni underwent a giant "eruption", with $\Delta V \sim 3 \mathrm{mag}$, and as a result lost, over an interval of only one decade, an amount of energy equal to its stored thermal energy. Such a major change cannot simply be the consequence of an unstable stratification in an outer layer, whose energy content is necessarily small. The most reasonable conclusion must be that the occasional major eruption is caused by an unsteady physical process located deep within the star, and that the stellar wind and the small fluctuations in visual magnitude are driven by the same mechanism. 
The following are the basic data for the present treatment:

$\begin{array}{ll}\text { Mass of P Cygni } & M=35 M_{\odot} \\ \text { Radius of photosphere } & r_{e}=5 \times 10^{12} \mathrm{~cm} \\ \text { Luminosity } & L=7.5 \times 10^{5} L_{\odot} \\ \text { Effective temperature (with fluctuations) } & T_{e}=20000 \mathrm{~K} \\ \text { Mass loss rate into the wind } & \dot{\mathcal{M}} \sim 10^{-5} M_{\odot} / \text { year }\end{array}$

The wind is fully ionized, certainly until shell formation occurs. The radiative acceleration outwards due to electron scattering is

$$
f_{r}=\kappa_{e} L / 4 \pi c r^{2}=2.7 \times 10^{27} / r^{2}
$$

at distance $r$ (expressed in CGS units) while the gravitational acceleration inwards is

$$
g=G M / r^{2}=4.7 \times 10^{27} / r^{2}
$$

and so the net inward acceleration becomes

$$
g_{\mathrm{eff}}=\mu / r^{2} \equiv 2 \times 10^{27} / r^{2} .
$$

The density in a wind with terminal speed $u_{\infty}$ is

$$
\rho=\frac{\dot{\mathcal{M}}}{4 \pi r^{2}\left(2 \mu / r+u_{\infty}^{2}\right)^{1 / 2}}
$$

at distance $r$, and the rate of recombinations per unit volume there is

$$
b n_{e}^{2}=\frac{b \rho^{2}}{m_{a}^{2}}=\frac{b \dot{\mathcal{M}}}{16 \pi^{2} m_{a}^{2} r^{3}\left(2 \mu+u_{\infty}^{2} r\right)}
$$

where $m_{a}$ is the mass per free electron.

The total rate of recombinations in the wind from the star out to some distance $r_{\text {ext }}$ is

$$
\begin{aligned}
S_{\text {rext }} & =4 \pi \int_{r_{*}}^{r_{\text {ext }}} b n_{e}^{2} r^{2} d r \\
& =\frac{b \dot{\mathcal{M}}^{2}}{8 \pi m_{a}^{2} \mu} \int_{r_{*}}^{r_{\text {ext }}} \frac{d r}{r\left(1+u_{\infty}^{2} r^{2} / 2 \mu\right)},
\end{aligned}
$$

where $b\left(=2 \times 10^{-13} \mathrm{~cm}^{3} \mathrm{~s}^{-1}\right)$ is the usual recombination coefficient in the ionized gas and $m_{a}=2 \times 10^{-24} \mathrm{gm}$. The integrated term contributes a logarithmic factor, of order unity. The coefficient outside equals $3.6 \times 10^{47} \mathrm{~s}^{-1}$, with the adopted numerical parameters. For comparison $\mathrm{P}$ Cygni produces photons in the Lyman continuum at a rate of some $10^{49} \mathrm{~s}^{-1}$, and so maintains full ionization, unless shell formation takes place.

The Ly c photons come from an atmosphere with temperature $20000 \mathrm{~K}$ and have an average energy $\left\langle\chi>\doteqdot 15.5 \mathrm{eV}=2.5 \times 10^{-11} \mathrm{erg}\right.$. They transfer outward momentum to the gas at a rate $b n_{e}<\chi>/ c$ and produce an acceleration

$$
f_{\mathrm{Ly}}=b n_{e}<\chi>/ m_{a} c \equiv \lambda \rho
$$


where

$$
\lambda \equiv b<\chi>/ m_{a}^{2} c=4 \times 10^{13} \mathrm{~cm}^{4} \mathrm{gm}^{-1} \mathrm{~s}^{-2} .
$$

In a steady wind, with speed $u$ at distance $r$,

$$
f_{\mathrm{Ly}}=\lambda \dot{\mathcal{M}} / 4 \pi u r^{2}
$$

and this acceleration is negligible compared with $g_{\text {eff }}$ unless

$$
\begin{aligned}
\lambda \dot{\mathcal{M}} / 4 \pi u & >\mu \\
\text { or } u<\lambda \dot{M} / 4 \pi \mu & \sim 10 \mathrm{~km} \mathrm{~s}^{-1} .
\end{aligned}
$$

Such slow winds do not occur in the present model for $\mathbf{P}$ Cygni.

\section{The Formation of Shells}

The wind will be treated as being cold. This approximation is reasonable enough since the wind speed is generally smaller by an order of magnitude than the (isothermal) sound speed $c_{i}$. As long as there are no collisions, that is in the time before the first shell forms, each part of the wind moves like an isolated stream in the effective gravitational field. The equation of motion integrates to give

$$
\frac{d r}{d t}=\left\{\frac{2 \mu}{r}+W(\tau)\right\}^{1 / 2}
$$

where $W(\tau)$ is twice the energy per unit mass of the stream and varies cyclically with the time of emission $\tau$. If $W$ is negative then the stream is gravitationally bound-until other effects take over. The solution of (6) is most conveniently expressed, for the purpose of this treatment, by writing

$$
t-\tau=\int_{0}^{r} \frac{d r}{\{2 \mu / r+W(\tau)\}^{1 / 2}} \equiv T(r, \tau) .
$$

The treatment that follows depends in detail on the sign of $W$ for that part of the wind on which the shell first forms, that is on whether it would have had enough energy, if left to itself, to escape from the reduced gravitational field of the star, or not. For the purpose of this calculation it is suitable to deal with the case where $W$ is positive.

Consider then how the function

$$
t \equiv \tau+T(r, \tau)
$$

depends on $\tau$, for progressively larger values of $r$. Close enough to the star the time of arrival, $t$, of a stream must vary monotonically with $\tau$, that is the streams arrive in the order that they were emitted, and $\partial t / \partial \tau$ is always positive. But with increasing distance $r$ the effect of the fluctuations in $W(\tau)$ becomes progressively more pronounced so that at one stage $\partial t / \partial \tau$ vanishes for just one instant in the cycle, and this condition defines the level $r_{s}$ and the time $t_{s}$ where the first shell forms; let the corresponding value of $\tau$ be $\tau_{s}$. Evidently $\partial t / \partial \tau$ at level $r_{s}$ is positive for $\tau\left\langle\tau_{s}\right.$ and $\tau>\tau_{s}$, and only vanishes at $\tau=\tau_{s}$. 
Consequently $\partial^{2} t / \partial \tau^{2}=0$ at $r=r_{s}, \tau=\tau_{s}$, and so the time of arrival at level $r_{s}$ of a stream emitted at time $\tau_{s}+\delta \tau$ is

$$
t_{s}+\delta t=t_{s}+\frac{1}{6} T_{3}(\delta \tau)^{3}
$$

where

$$
T_{3} \equiv \partial^{3} T / \partial \tau^{3}
$$

Let $V_{s}$ be the speed of the shell at the time when it forms. The stream which left the star at time $\tau_{s}+\delta \tau$ lags by a distance

$$
V_{s} \delta t=\frac{1}{6} V_{s} T_{3}(\delta \tau)^{3}
$$

at time $t_{s}$, but its speed is larger by an amount

$$
\Delta V=\frac{(d W / d \tau) \delta \tau}{V_{s}}
$$

It will therefore catch up the newly formed shell at time $t_{s}+\Delta t$, where

$$
\begin{aligned}
\Delta t \equiv \frac{V_{s} \delta t}{\Delta V} & =\frac{V_{s}^{2} T_{3}}{6 d W / d \tau}(\delta \tau)^{2} \\
& =\Lambda(\delta \tau)^{2}
\end{aligned}
$$

say.

The mass per unit area that has accumulated on each side of the shell is, at time $t_{s}+\Delta t$,

$$
\Sigma=\frac{\dot{\mathcal{M}} \delta \tau}{4 \pi r_{s}^{2}}=\frac{\dot{\mathcal{M}}}{4 \pi r_{s}^{2}}\left(\frac{\Delta t}{\Lambda}\right)^{1 / 2}
$$

and its rate of arrival, per unit area, is

$$
\dot{\Sigma}=\frac{\dot{\mathcal{M}}}{8 \pi r_{s}^{2}(\wedge \Delta t)^{1 / 2}} .
$$

The stream that arrives at time $t_{s}+\Delta t$ has a speed $\Delta V$ relative to the shell (see relation (11)). The pressure on each side of the shell is therefore

$$
P=\dot{\Sigma} \Delta V=\frac{3}{4 \pi} \frac{\dot{\mathcal{M}}(d W / d \tau)^{2}}{r_{s}^{2} V_{s}^{3} T_{3}}
$$

Since the (isothermal) sound speed in the shell is assumed to be $c_{i}(=10 \mathrm{~km} / \mathrm{s})$ the density of the gas in the shell is

$$
\rho=\frac{3}{4 \pi c_{i}^{2}} \frac{\dot{\mathcal{M}}(d W / d \tau)^{2}}{r_{s}^{2} V_{s}^{3} T_{3}}
$$

and the acceleration, due to the pressure of Ly $\mathrm{c}$ photons, is $\lambda \rho$.

This formalism has now to be reconciled with the observations of shells in P Cygni, reported by de Groot (1969), Markova and Luud (1983), Markova and Kolka (1984) and 
Markova (1986). The period between the creation of successive shells is about two months, the shells have speeds of about $100 \mathrm{~km} \mathrm{~s}^{-1}$ when they first appear, and over a period of seven months or so they accelerate to $220 \mathrm{~km} \mathrm{~s}^{-1}$. The typical acceleration is therefore about $0.6 \mathrm{~cm} \mathrm{~s}^{-2}$.

The first step is to assume a value for $r_{s}$, the radial distance at which a shell forms. The value to be used for $d W / d \tau$, in equation (16), can then be calculated by noting that

$$
V_{s}^{2}=\frac{2 \mu}{r_{s}}+W\left(\tau_{s}\right)
$$

and that a necessary condition for shell formation is that

$$
\begin{aligned}
0=1 & +\frac{\partial T}{\partial \tau} \equiv 1+\frac{\partial}{\partial \tau} \int_{0}^{r_{s}} \frac{d r}{[2 \mu / r+W(\tau)]^{1 / 2}} \\
= & 1-\frac{d W / d \tau}{2} \int_{0}^{r_{s}} \frac{d r}{\left(2 \mu / r+V_{s}^{2}-2 \mu / r_{s}\right)^{3 / 2}} .
\end{aligned}
$$

The value of $T_{3}$ cannot be determined unless the form of variation of $W(\tau)$ is known. To make progress, therefore, set

$$
W(\tau)=\bar{W}(1+\epsilon \cos \omega \tau)
$$

so that the fluctuation in the specific energy content is sinusoidal, and set $2 \pi / \omega$ equal to two months, the interval between the appearance of successive shells. With this simplification it becomes possible, but is rather tedious, to calculate $T_{3}$, and hence $\rho$ and $\lambda \rho$ in terms of assumed values of $\dot{\mathcal{M}}$ and $r_{s}$. The initial acceleration of the shell is then the resultant of an acceleration $\lambda \rho$ outwards and $\mu / r_{s}^{2}$ inwards, towards the star, giving that

$$
\lambda \rho=\frac{\mu}{r_{s}^{2}}+0.6
$$

Representative results are given in Table 1 below, for three assumed values of the radial distance $r_{s}$.

These results help determine the value of $\dot{\mathcal{M}}$ appropriate for shell formation at each radial distance $r_{s}$. The shell has a mass which ultimately grows to $M_{s}=2 \pi \dot{\mathcal{M}} / \omega$ and, when it is put under pressure $P$, the number of Ly c photons required, per second, to keep it ionized is

$$
\begin{array}{lcccl}
\multicolumn{4}{c}{S_{\text {req }}=\frac{b \mathcal{M}{ }_{s} P}{m_{a}^{2} c_{i}^{2}}=\frac{2 \pi b \dot{\mathcal{M}} P}{\omega m_{a}^{2} c_{i}^{2}}} \\
& & & & \\
r_{s} & 5 \times 10^{13} & 10^{14} & 1.5 \times 10^{14} & \mathrm{~cm} \\
P / \dot{\mathcal{M}} & 2.64 \times 10^{-22} & 1.86 \times 10^{-23} & 3.79 \times 10^{-24} & \mathrm{~cm}^{-1} \mathrm{~s}^{-1} \\
T_{3} & 2.68 \times 10^{-12} & 1.64 \times 10^{-12} & 1.25 \times 10^{-12} & \mathrm{~s}^{-2} \\
f=\mu / r_{s}^{2}+0.6 & 1.40 & 0.80 & 0.69 & \mathrm{~cm} \mathrm{~s}^{-2} \\
\rho=f / \lambda & 3.5 \times 10^{-14} & 2 \times 10^{-14} & 1.73 \times 10^{-14} & \mathrm{gm} \mathrm{cm}^{-3} \\
P=\rho c_{i}^{2} & 3.5 \times 10^{-2} & 2 \times 10^{-2} & 1.73 \times 10^{-2} & \text { dyne cm } \\
& & & &
\end{array}
$$

Table 1: 


$\begin{array}{lcccl}r_{s} & 5 \times 10^{13} & 10^{14} & 1.5 \times 10^{14} & \mathrm{~cm} \\ \dot{\mathcal{M}} & 1.33 \times 10^{20} & 1.08 \times 10^{21} & 4.56 \times 10^{21} & \mathrm{gm} \mathrm{s}^{-1} \\ S_{\text {req }} & 1.20 \times 10^{48} & 5.65 \times 10^{48} & 2.07 \times 10^{49} & \mathrm{~s}^{-1} \\ 1.2 \times 10^{7} \dot{\mathcal{M}} & 1.60 \times 10^{27} & 1.30 \times 10^{28} & 5.47 \times 10^{28} & \mathrm{gm} \mathrm{cm} \mathrm{s}^{-2} \\ S_{*}\langle\chi>/ c & \leftarrow & 8.33 \times 10^{27} & \rightarrow & \mathrm{gm} \mathrm{cm} \mathrm{s}\end{array}$

Table 2:

Finally the momentum required per unit time to speed up all the shells by $120 \mathrm{~km} / \mathrm{s}$ is $1.2 \times 10^{7} \dot{\mathcal{M}}$ : the momentum per unit time carried by the Lyman continuum flux is $S_{*}\langle\chi\rangle / c$. Table 2 shows the relation between these quantities.

If $r_{s}$ were as small as $5 \times 10^{13} \mathrm{~cm}$ then the number of Lyman continuum photons needed to keep the innermost shell ionized would be about one tenth of the number produced by $P$ Cygni. This would conflict with the observation that only three or four shells are visible at any one time. On the other hand $\mathrm{P}$ Cygni does not produce enough Ly c photons to keep even one shell ionized, for the case of $r_{s}=1.5 \times 10^{14} \mathrm{~cm}$. When $r_{s}=10^{14} \mathrm{~cm}$ the inner shell soaks up about half the available ionizing radiation, which seems about right, but the Lyman continuum flux does not provide quite enough radiation pressure to accelerate the flow. Interpolation of the data in the table indicates that momentum balance is achieved for $\dot{\mathcal{M}}=6.8 \times 10^{20} \mathrm{gm} \mathrm{s}^{-1}$ and $r_{s}=8.6 \times 10^{13} \mathrm{~cm}$, and that then $S_{\text {req }}=4.2 \times{ }^{48} \mathrm{~s}^{-1}$ so that the innermost shell soaks up about two-fifths of the available Lyman continuum photons.

\section{Discussion}

It is interesting that the stream on which the shell forms takes about $2 \frac{1}{2}$ months to rise to radial distance $r_{s}$. In fact it is only to be expected that the dynamics of the wind should determine the interval between episodes of shell formation since P Cygni itself does not possess any likely modes of oscillation that have a period which is even of the right order of magnitude. The observed photometric variations of luminous blue variables look rather like a manifestation of random noise. The real question is therefore why and how the flow selects the frequencies at which shells appear from the broad band disturbances introduced at the star. A paper to follow (Kahn 1989) will address this problem and also deal more thoroughly with the physical nature of the flow.

\section{References}

de Groot, M.: 1969, Bull. Astr. Inst. Netherlands, 20, 235.

Lamers, H. J. G. L.M.: 1987 in Instabilities in Luminous Early Type Stars, eds. Lamers, H. G. J. L. M. and de Loore, C. W. H., Reidel, Dordrecht, p.99.

Markova, N.: 1986, Astron. Astrophys., 162, L3.

Markova, N. and Kolka, I.: 1984, Astrofiz., 20, 250.

Markova, N. and Luud, L.: 1983, Publ. Tartu Astrophys. Obs., 32, 55. 


\section{DISCUSSION}

Davidson: Lyman alpha?

Kahn: Yes, $L \alpha$ becomes important when the shell reaches a place where the $L c$ runs out. The $L \alpha$ photons press on the neutral $\mathrm{H}$-atoms there.

Appenzeller: Are the shells that you described subject to Rayleigh-Taylor instabilities and could such instabilities modify or destroy the shells?

Kahn: Rayleigh-Taylor instabilities very likely become important in the outer, less ionized layers that I just mentioned, where $L \alpha$ radiation begins to push hard on the neutral hydrogen.

Sreenivasan: You said that the first shell forms way out in the supersonic region of the flow. What happens to the bits of the shell that fall back? Shouldn't they be lifted upward by the supersonic wind that is coming off the star? Is there a Rayleigh-Taylor instability as a result?

Kahn: None of the material in the shell ought to fall back, because at the high density prevalent in the shell the pressure in the Lyman continuum is too strong to allow it to do so.

Kudritzki: I am wondering how your treatment would have to be modified in order to include the effects of radiation pressure in metal lines.

Kahn: In principle the process of shell formation should be the same, provided that there are no important effects dependent on the density of the gas or on the velocity gradient. If there are such effects, then a drastic re-think is needed.

De Groot: The light curve of $\mathrm{P}$ Cygni shows maxima and minima on a timescale of 40 days, the same as a value you mentioned in your talk. Maxima and minima occur at various brightness levels, indicative of the clumpiness or anisotropy of your shell formation process.

Kahn: It seems to me that random fluctuations are superposed on a steady wind in the case of P Cygni. The dynamics of the wind rising from the star acts like a filter that picks out the effects of fluctuations in the right frequency range.

Lamers: The calculations described by Kahn may be too simple for the instabilities in the winds of O-stars (as modeled, e.g. by Owocki and colleagues), but they may be well suited for the LBV winds, in particular for P Cygni. IUE observations (Lamers et al. 1986) show that the ionization of the shells is lower than in the quiescent wind. Kudritzki has shown that the effective radiation pressure increases drastically when the ionization drops from Fe III to Fe II, etc. This is similar to Kahn's assumptions.

Conti: Why is there only one P Cygni? If it is so easy for an LBV to eject multiple shells, why don't more stars show this?

Lamers: P Cygni is not the only LBV showing these features. All LBV's have multiple absorption components in their line profiles (visual and UV wavelengths) if you look carefully, but they have been followed in time only for P Cygni. So I suspect that most LBV's have variable shells like P Cygni. I have the impression that these variable components occur mainly during minimum or quiescent states. 


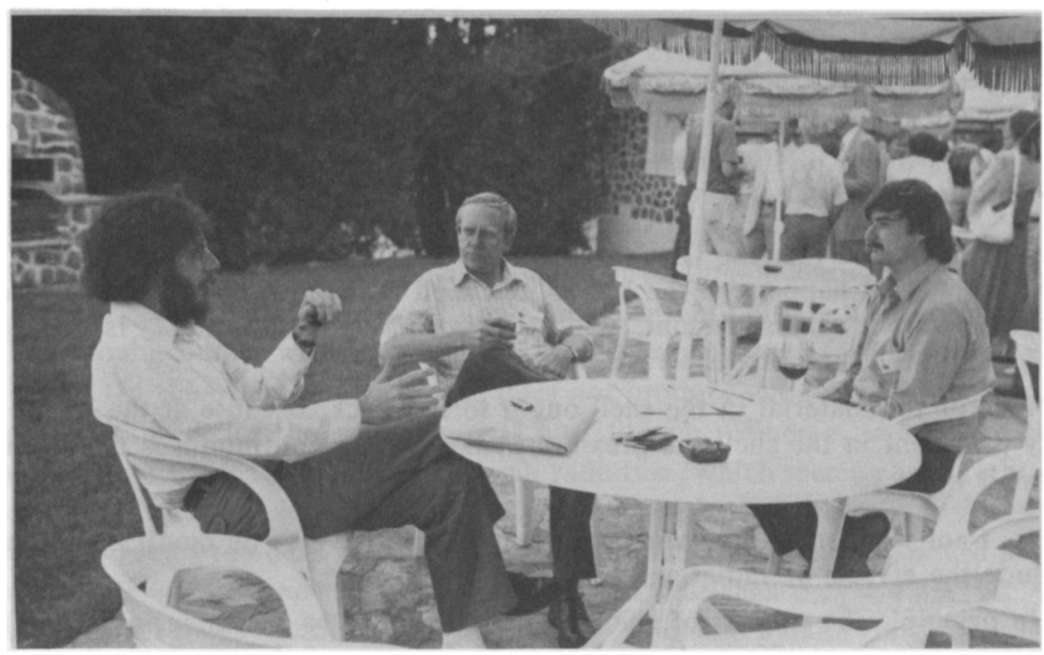

Shore, Cassinelli, Hillier 